\title{
Fiberoptic Microtransducer Pressure Technology: Urodynamic Implications
}

\author{
William D. Belville, Stanley J. Swierzewski III, Gary Wedemeyer, and \\ Edward J. McGuire
}

Department of Surgery, Section of Urology, University of Michigan Medical Center, Ann Arbor, Michigan

\begin{abstract}
The FST 200 is a novel commercially available pressure measurement system that combines microtechnology and fiberoptics and is particularly well suited for invasive urodynamic studies. Pressure recording with this system is highly accurate and reproducible. The pressure curves obtained parallel those from standard water cystometry. Being small and portable, the system lends itself to invasive transurethral monitoring in the privacy of an examination room. Additionally, the 5F catheter size allows the performance of leak point pressures and pressure/flow voiding studies with minimal urethral stenting artifact often seen with larger catheters. The technical aspects of this system are presented.

(c) 1993 Wiley-Liss, Inc.
\end{abstract}

Key words: fiberoptic, microtransducer, urodynamic

\section{INTRODUCTION}

There are many systems in clinical use for the urodynamic investigation of vesicourethral function and dysfunction. We have used a Bard triple lumen catheter, and fluoroscopy for video urodynamics for these investigations for 20 years [Woodside and McGuire, 1985]. That system is valuable for research studies and in the diagnosis of complicated causes of incontinence. Based on the data obtained with fluoroscopically guided multichannel studies, it is apparent that, in most clinical situations, these are not required. Measurement of bladder compliance, abdominal and bladder pressures required to cause leakage, and pressure flow studies are accurate and useful and can be done without multichannel capability. These studies can be related to long-term outcome with respect to treatment selected, and treatment predicated on these studies can be shown to be effective [Wang et al., 1988; Schäfer et al., 1989].

Video urodynamics requires the examiner to be present, usually with several other individuals, which makes the procedure less than "natural," and often prevents accurate reproduction of the existent clinical problem. When evaluating leak point

Received for publication June 16, 1992; accepted August 11, 1992.

Address reprint requests to William D. Belville, Department of Surgery, Section of Urology, University of Michigan Medical Center, 1500 E. Medical Center Drive, Ann Arbor, MI 48109. 
pressures (bladder or abdominal), or during pressure flow studies, catheter size has an influence on flow characteristics, urethral resistance, and external sphincter activity, which is difficult to quantitate.

We initially applied this new fiberoptic pressure measuring system in spinal cord patients for the simultaneous measurement of bladder compliance, during flexible cystoscopy, with the patient in his/her wheelchair. We quickly adapted the system for pressure flow studies (PFS) and for leak point pressures (LPP); in so doing, we compared our results with this system with traditional methods using external transducers.

\section{MATERIALS AND METHODS}

The FST 200 Urodynamic pressure monitoring system (FiberOptic Sensor Technologies, Ann Arbor, MI) is an offshoot of fiberoptic microtransducer technology developed during the 1980 s for invasive cardiovascular studies. The system includes a $5 \mathrm{~F}$ fiberoptic pressure catheter, a pressure monitor and interface cable which consists of technology similar to electronic "microtip" catheters but, unlike electronic catheters, the fiberoptic catheter is not fragile and can be used multiple times. The FDA has approved the catheter for multiple use and, with proper handling, can be expected to perform as many as 50 studies.

The system incorporates a sensor that is immune to electrostatic discharge, electromagnetic interference, and pulses. Unlike electronic catheters, this system uses light to transmit the pressure signal and thus poses no risk of electrical current leakage. Furthermore, it provides precise pressure measurements with high-resolution waveforms. Direct sensor tip measurement eliminates damping effects and movement artifact inherent in fluid-based systems or catheter-mounted transducers. These systems, like catheter-mounted transducers, are unsuitable for urethral pressure measurements [Schäfer, 1989]. The FST 200 system was contrasted to standard water cystometry (a Sorenson disposable pressure transducer, a Hewlett Packard recorder, a pressurized uroflow system with a zero setting at the symphysis level), using an identical filling rate of $100 \mathrm{ml} / \mathrm{min}$.

\section{TECHNOLOGY}

The FST 200 transmits light via an internal light-emitting diode (LED), to the "FST CATH-UD' catheter optical connector (Fig. 1). The light travels down a fiberoptic wave guide housed inside the catheter tubing. The light reflects off the inner face of the reflective titanium diaphragm housed at the distal tip of the catheter. Movement of the diaphragm is proportional to any external pressure applied to it, such as bladder pressure. The higher the pressure exerted, the closer the diaphragm moves to the end face of the optical fiber. As the diaphragm moves closer to the fiber end face, the intensity of the reflected light that the fiber sees increases; this change in light intensity is transmitted back down the fiber to the FST 200 Monitor. (Note that the catheter sensor is enclosed and hermetically sealed.) The monitor then converts the intensity reading to an electronic current via a photodiode. The electronic signal is processed and converted to pressure measurement units, $\mathrm{cm} \mathrm{H}_{2} \mathrm{O}$ (see the explanation under Calibration Cycle) for liquid crystal display (LCD) on the FST 200 front panel. Pressure readings are sent to the FST 200 interface output for continuous 


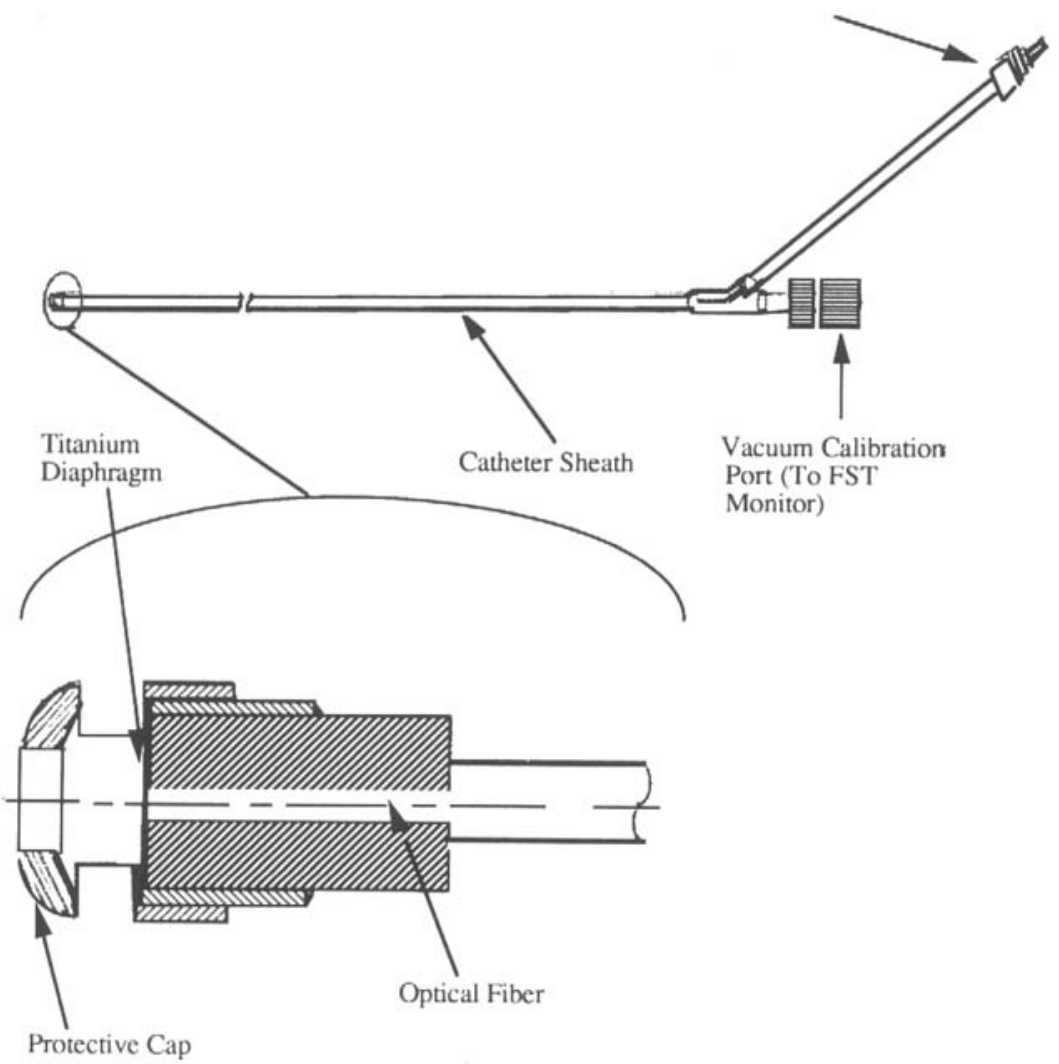

Fig. 1. Fiberoptic microtransducer. Arrow at top indicates the optical fiber connector (to FST monitor).

waveform display on any monitor that conforms to Association for the Advancement of Medical Instrumentation (AAMI) specification for transducer interchangeability including, but not limited to, Dantec, Lifetech, etc.

\section{Calibration Cycle}

Because of properties intrinsic to the design of the FST fiberoptic pressure sensor, a calibration of the system is required before each use with the catheter positioned at the level of the symphysis. Calibration is accomplished automatically after connecting the catheter to the FST 200 Monitor and pressing the monitor calibration button. During the calibration cycle, the FST 200 pulls a negative pressure of approximately $400 \mathrm{~cm} \mathrm{H}_{2} \mathrm{O}$ on the "vacuum" port connected to the catheter's proximal "vacuum" luer connector. This negative pressure is then exerted, via the catheter tube, on the internal side of the diaphragm, pulling the diaphragm close to the fiber end-face. The pressure is then slowly released inside the FST 200 Monitor. As the pressure is released, the titanium diaphragm slowly relaxes and returns to its zero position. As the diaphragm returns to its zero position, the FST 200 monitors the change in light intensity reflection of the diaphragm and compares those signals with pressure readings using a National Institute of Standards and Technologies traceable 


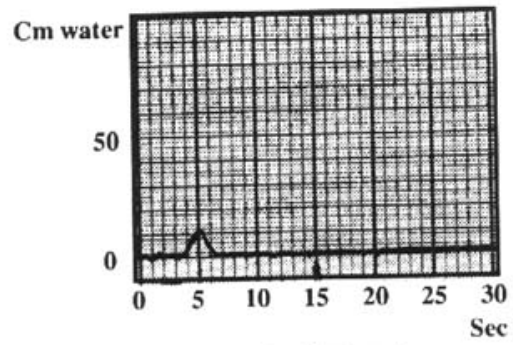

Fiber Optic Transducer

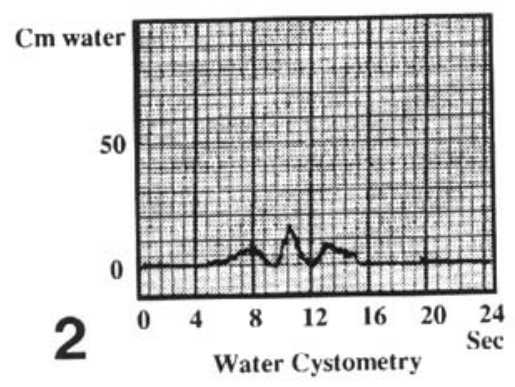

Fig. 2. Patient cough at initiation of study for illustrative purposes.
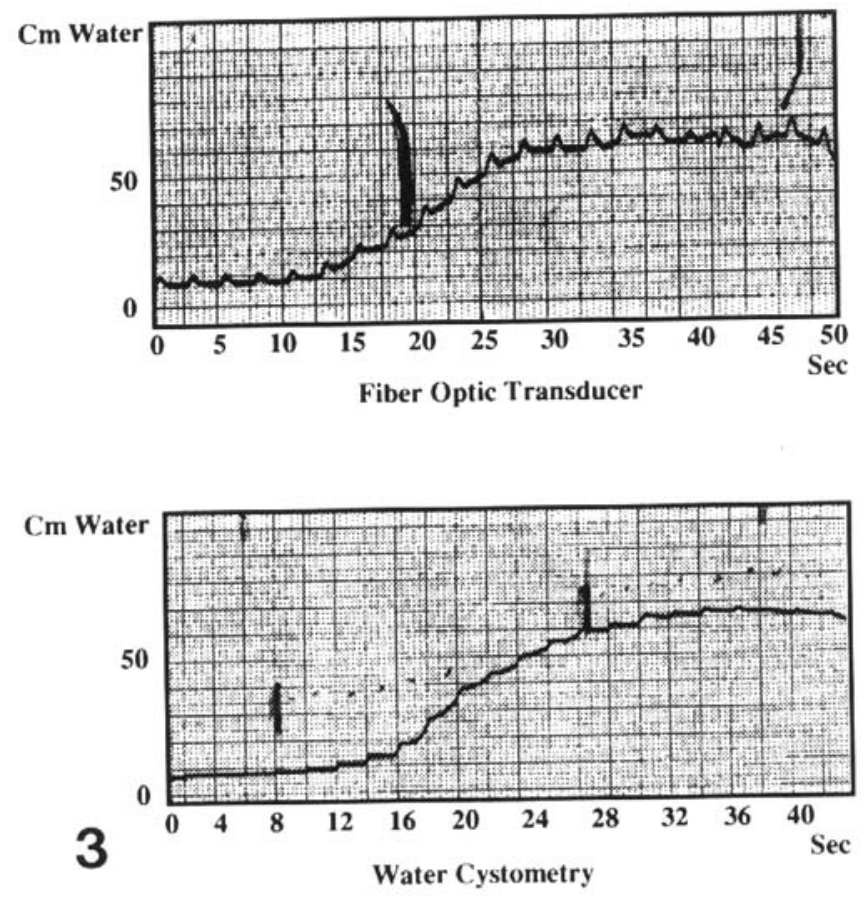

Fig. 3. Non-compliant bladder. 

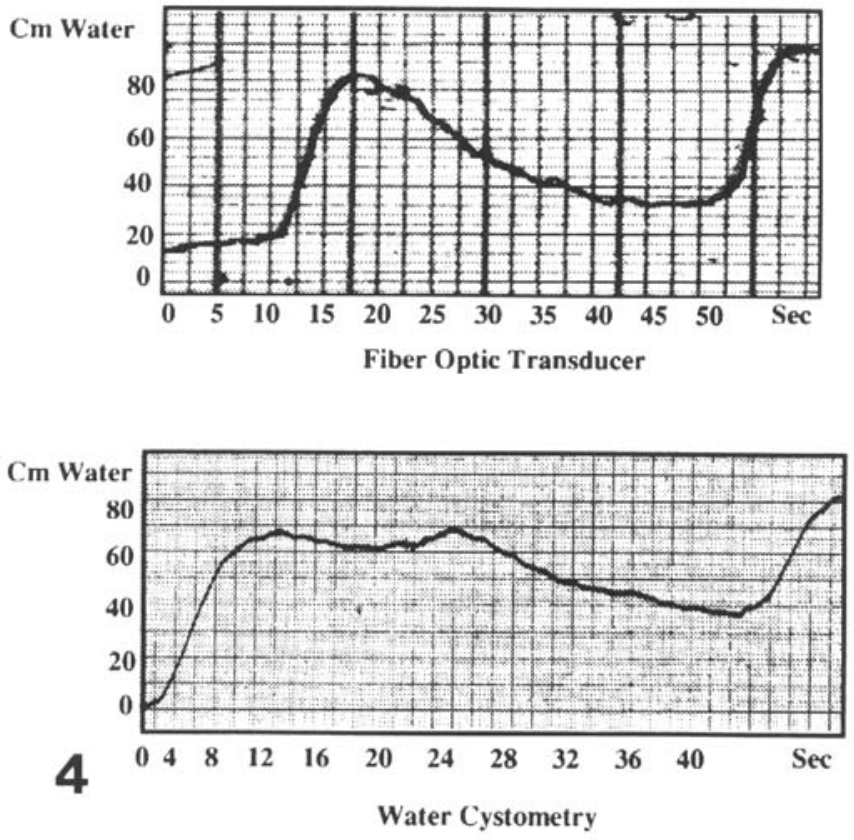

Fig. 4. Detrusor instability.
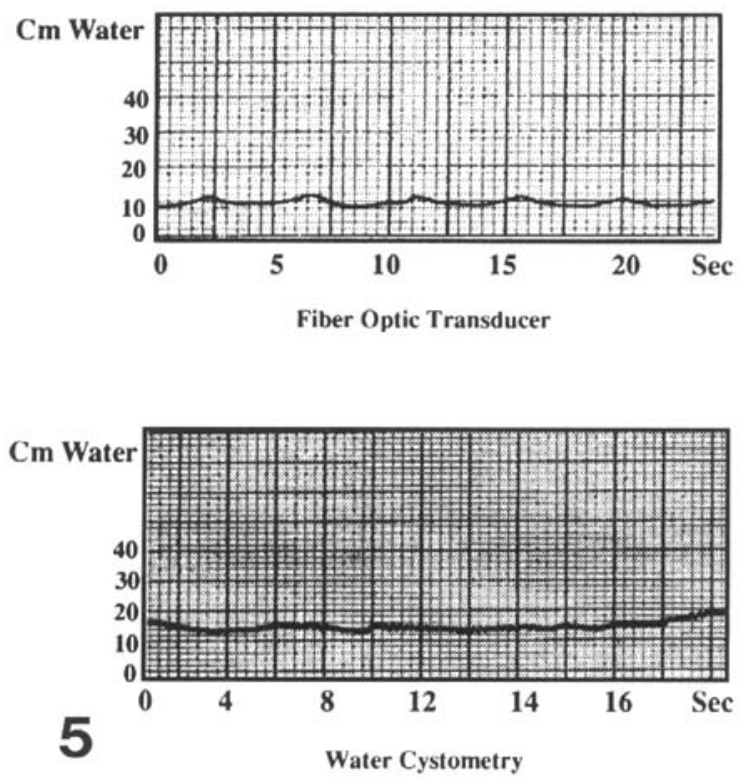

Fig. 5. Areflexic bladder. 


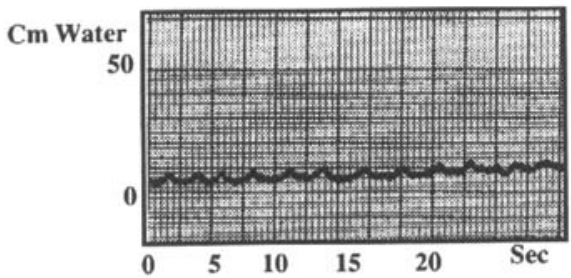

Fiber Optic Transducer

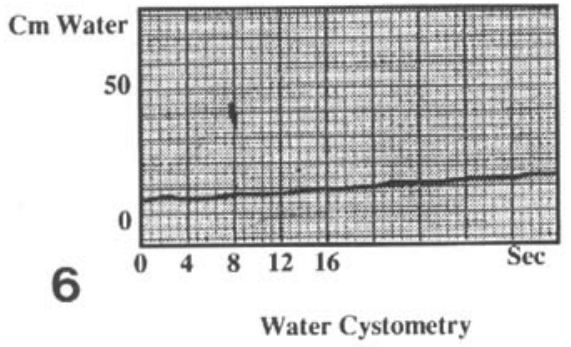

Fig. 6. Respiratory excursions evident with fiberoptic transducer.

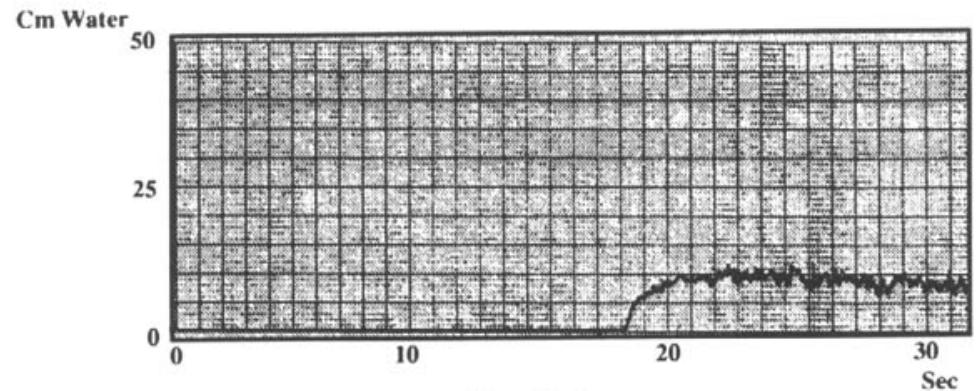

Flow Rate

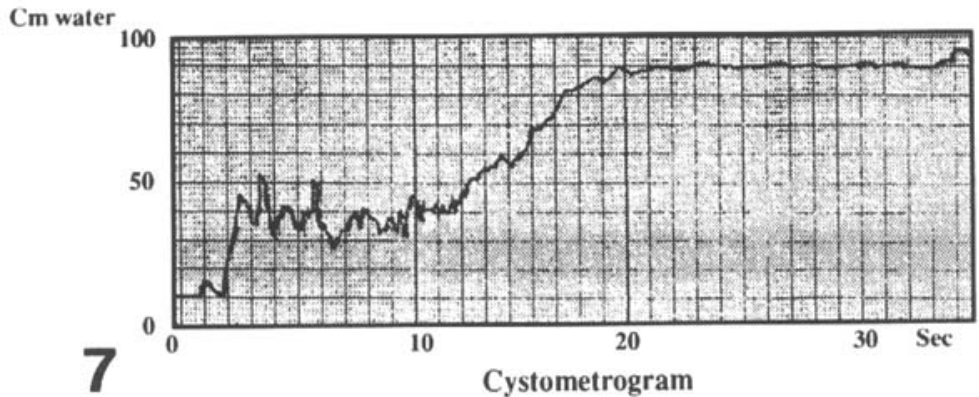

Fig. 7. Synchronous pressure flow study. Flow does not begin until bladder pressure exceeds $80 \mathrm{~cm}$ of water. 
pressure transducer that is internal to FST 200 Monitor. The FST 200's internal microprocessor then constructs a "look-up" table consisting of comparison data points between the catheter diaphragm position (or light intensity) and pressure readings taken from the internal transducer. The FST 200 then stores this table in internal memory (RAM).

The calibration cycle takes $15 \mathrm{~s}$ to complete; the clinician is then ready to introduce the catheter and to start to take pressure readings. As pressures are being exerted on the catheter diaphragm, the FST 200 will continuously convert the light intensity changes to a linearized pressure reading using the look-up table as its conversion factor. The FST system can resolve pressure readings to sub-cm $\mathrm{H}_{2} \mathrm{O}$ levels with a frequency response of $50 \mathrm{~Hz}$, thereby yielding very accurate highresolution pressure readings and waveforms. The FST system has the additional capability of being easily recalibrated to zero at any time. This insures the user that the system is accurate even after multiple catheter use.

\section{RESULTS}

The pressure-volume curves obtained in more than 20 different clinical situations were compared to standard water cystometry with identical rates of fill ( 100 $\mathrm{ml} / \mathrm{min}$ ) done immediately prior to fiberoptic measurements. In all cases, the curves were identical. At identical bladder volumes, the mean range of maximum bladder pressures recorded for the FST system was 39.3-42.9 versus a mean of 39.5-43.1 $\mathrm{cm} \mathrm{H}_{2} \mathrm{O}(\mathrm{N}=20)$. The tracings from pressure flow studies were especially easy to record.

Figures 2-7 illustrate combined results in several clinical situations. Figure 2 demonstrates a patient's cough at the start of the studies. In Figure 3, the slow climbing curve of a noncompliant bladder is seen. Figure 4 shows a uninhibited contraction, and Figure 5 demonstrates a areflexic bladder. The sensitivity of the microtransducer is illustrated in Figure 6, where respiratory excursions, not recorded with water column technology are seen. Figure 7 shows a simultaneous pressure flow study in which bladder pressure is seen to rise to $>90 \mathrm{~cm} \mathrm{H}_{2} \mathrm{O}$ with a poor flow rate.

\section{DISCUSSION}

Initially, we evaluated the suitability of this unique system for synchronous cystometry/cystoscopy via a flexible cystoscope in our spinal cord injury patients. We were most pleased with the durability, portability, and accuracy when contrasted to our standard external transducer method. Therefore, we adapted it for other indications. While the system lends itself to abdominal/rectal pressure measurement, we have had, over the years, a disappointing experience with rectal catheters and have all but abandoned their use.

It soon became clear that one $10 \mathrm{~F}$ urethral instrumentation would permit the performance of cystometry followed by a pressure flow study simply by removing the $10 \mathrm{~F}$ introducing catheter over the indwelling $5 \mathrm{~F}$ microtransducer and allowing the patient to void. This application makes the system adaptable to most uroflow equipment and permits sophisticated PFS in the privacy of an examination or wash room, allowing more natural micturition. Additionally, the $5 \mathrm{~F}$ size reduces the significance of the stent artifact from urethral catheterization especially in regard to abdominal or 
bladder LPP. Lastly, the $10-\mathrm{ms}$ response time is in contrast to the $25 \mathrm{~ms}$ with a water column, thus reducing dampening and reflecting a "real-time" pressure.

\section{CONCLUSIONS}

This system provides accurate intravesical pressure recordings useful for cystometry, bladder and abdominal leak point pressures, and pressure flow studies. The FST system allows for avoidance of a columnated system with its slow response time, and requirement for bulky immobile fixed equipment. Also, the portability of the FST system provides for collection of real-time data, in a private setting. A planned reduction of the catheter to $3 \mathrm{Fr}$ size and miniaturization of the monitor will further enhance ambulatory urodynamics capability as well as further open endoscopic monitoring of the upper urinary tract. This system is a welcome addition in the urodynamic equipment armamentarium.

\section{ACKNOWLEDGMENTS}

The authors wish to express our heartfelt appreciation to Ms. Carrie Griffith for her patience, diligence, and editorial input in the preparation of this manuscript.

\section{REFERENCES}

Schäfer W (1989): Editorial Comment to Benson, TJ. Clinical significance of axial variations in female urethral pressure probes. Neuroruol Urodyn 8:491-504.

Schäfer W, Rübben H, Noppeney R, Deutz S-J (1989): Obstructed and unobstructed prostatic obstruction. A plea for urodynamic objectivation of bladder outflow obstruction in benign prostatic hyperplasia. World J Urol 6:198-203.

Wang SC, McGuire EJ, Bloom DA (1988): A bladder pressure management system for myelodysplasiaClinical outcome. J Urol 140:1499-1502.

Woodside JR, McGuire EJ (1985): Clinical evaluation of new urodynamic catheter. Urodynamics 25: 95-96. 